\title{
LV-EV-3
}

\section{Laparoscopic liver resection for locally advanced tumor}

\author{
YOUNGROK CHOI*
}

Seoul National University College of Medicine, Seoul, Korea

Lecture: The Barcelona Clinic Liver Cancer (BCLC) staging system categorizes a patient with hepatocellular carcinoma (HCC) according to the characteristics of the tumor, the liver disease, and the performance status of a patient. However, the BCLC algorithm is too conservative in offering resection for advanced HCC, including the locally advanced tumors. As a result, this system recommends systemic therapy for patients with advanced HCC as the first-line treatment with Sorafenib because the portal vein tumor thrombus is often interpreted as metastatic disease. Besides, there are still some uncertainties to categorize HCC with the bile duct invasion and surgical role also indefinite for advanced HCC.

A number of international guidelines have excluded the role of surgery for these patients. Nevertheless, multiple studies have found a clear benefit for selected patients and have questioned the appropriateness of published recommendations, in particular concerning the role of liver resection.

Usually, en bloc resection of the tumor together with affected structures might show good oncological outcomes \& removal of residual microscopic foci. Even though patients with advanced HCC are at high risk of treatment failure, recurrence, and mortality, outcomes of liver resection should be examined and weighed against the lack of any other potentially curative option.

Video clips show the 3 cases of laparoscopic liver resection for locally advanced HCC including, huge HCC, HCC with bile duct invasion, and down-staged HCC after TARE. We applied the recently developed laparoscopic surgical skill to remove the advanced HCC to get fewer postoperative complications, less postoperative pain, and shorter hospital stays. 